\title{
Final Report: Towards Optimal Petascale Simulations (TOPS), ER25785
}

\author{
Daniel R. Reynolds ${ }^{1}$ \\ April 15, 2011
}

\begin{abstract}
Multiscale, multirate scientific and engineering applications in the SciDAC portfolio possess resolution requirements that are practically inexhaustible and demand execution on the highest-capability computers available, which will soon reach the petascale. While the variety of applications is enormous, their needs for mathematical software infrastructure are surprisingly coincident; moreover the chief bottleneck is often the solver. At their current scalability limits, many applications spend a vast majority of their operations in solvers, due to solver algorithmic complexity that is superlinear in the problem size, whereas other phases scale linearly. Furthermore, the solver may be the phase of the simulation with the poorest parallel scalability, due to intrinsic global dependencies. This project brings together the providers of some of the world's most widely distributed, freely available, scalable solver software and focuses them on relieving this bottleneck for many specific applications within SciDAC, which are representative of many others outside. Solver software directly supported under TOPS includes: hypre, PETSc, SUNDIALS, SuperLU, TAO, and Trilinos. Transparent access is also provided to other solver software through the TOPS interface.
\end{abstract}

The primary goals of TOPS are the development, testing, and dissemination of solver software, especially for systems governed by PDEs. Upon discretization, these systems possess mathematical structure that must be exploited for optimal scalability; therefore, application-targeted algorithmic research is included. TOPS software development includes attention to high performance as well as interoperability among the solver components. Support for integration of TOPS solvers into SciDAC applications is also directly supported by this proposal.

The role of the UCSD PI in this overall CET, is one of direct interaction between the TOPS software partners and various DOE applications scientists - specifically toward magnetohydrodynamics (MHD) simulations with the Center for Extended Magnetohydrodynamic Modeling (CEMM) SciDAC and Applied Partial Differential Equations Center (APDEC) SciDAC, and toward core-collapse supernova simulations with the previous Terascale Supernova Initiative (TSI) SciDAC and in continued work on INCITE projects headed by Doug Swesty, SUNY Stony Brook. In addition to these DOE applications scientists, the UCSD PI works to bring leading-edge DOE solver technology to applications scientists in cosmology and large-scale galactic structure formation.

Unfortunately, the funding for this grant ended after only two years of its five-year duration, in August 2008, due to difficulties at DOE in transferring the grant to the PI's new faculty position at Southern Methodist University. Therefore, this report only describes two years' worth of effort.

1 TOPS PI at the Unversity of California, San Diego. Current address is SMU Department of Mathematics, PO Box 750156, Dallas, TX 75275, reynolds@smu.edu. 
1.Scalable Implicit Solvers for Fusion Plasmas 3

a. Problem/collaboration introduction 3

b. Ideal MHD preconditioner description 3

c. Results 4

d. Solver ramifications on solenoidal MHD constraint 5

$\begin{array}{ll}\text { e. Scalability improvements } & 6\end{array}$

f. Extensions to resistive MHD 7

g. Extensions to shaped plasmas

h. Talks and Papers 9

2.Implicit Solvers for Coupled Multiphysics Simulations Involving Shocks 9

a. Problem/collaboration introduction 9

$\begin{array}{lr}\text { b. Computational approach } & 10\end{array}$

$\begin{array}{ll}\text { c. Talks and Papers } & 10\end{array}$

3.Implicit Solvers for Large-Scale Simulations of Cosmological 11

Reionization

a. Problem/collaboration introduction 11

b. Numerical methods 11

$\begin{array}{ll}\text { c. Results } & 11\end{array}$

d. Talks and Papers 12

4.Presentations, Workshops and Manuscripts Resulting From This Work 13 


\section{Scalable Implicit Solvers for Fusion Plasmas}

\section{Problem/collaboration introduction:}

Plasma physics simulations are a key component to scientific understanding of both fusion plasmas and atmospheric processes. A standard model in both regimes is that of magnetohydrodynamics (MHD), which couples the compressible Euler equations for gas dynamics with the low-frequency Maxwell equations for evolution of electromagnetic fields. The MHD equations exist in three basic variants, each with increasing physical realism: ideal MHD, visco-resistive MHD, and extended MHD. The basic ideal MHD model is hyperbolic in type, but exhibits extreme numerical stiffness due to the presence of non-energetic but rapid fastmagnetosonic and Alfven waves. The latter models increase the numerical stiffness, due to the introduction of diffusion in the visco-resistive model, and the introduction of dispersive wave in the extended model. As such, large-scale simulations of these equations, especially for fusion plasmas, require the use of implicit time methods for computational efficiency.

It is in this context that our work resides, namely in the development of implicit solvers of optimal complexity for solution of PDE systems modeling fusion plasmas. As with most modern approaches to fully implicit solvers, we utilize an inexact Newton-Krylov method, wherein each time step of the evolution requires the solution of a large-scale nonlinear problem. The inexact Newton algorithm in turn reduces this nonlinear problem into a sequence of large-scale linear solves. The Krylov method then reduces each linear solve to a sequence of highly efficient matrix-vector products. However, for true scalability of this overall approach, a preconditioner must be used to accelerate convergence of the Krylov iteration as the overall problem size increases.

The role of this preconditioner is to provide an approximate solution approach to linearizations of the original nonlinear partial differential equation (PDE) operator. For a preconditioning approach to enable effective utilization of modern large-scale computing resources, the preconditioner must simultaneously provide a good approximation to the original PDE, while also allowing a high computational efficiency, and optimal parallel scalability.

\section{Ideal MHD preconditioner description:}

In this work, we have completed the mathematical derivation, theoretical analysis and computational testing of a new preconditioning strategy aimed at stiff, ideal MHD problems, in collaboration with Princeton Plasma Physics scientist Ravi Samtaney and Lawrence Livermore National Laboratory scientist Carol Woodward.

The preconditioning approach that we have developed aims to alleviate stiffness due to the fastest waves inherent in the MHD formulation. This works through a multi-stage process:

(1) Directionally split the implicit Newton system into separate systems for each Cartesian direction.

(2) Project the linear implicit Newton systems that are written in terms of conserved variables into characteristic variables, effectively splitting the coupled implicit system into separate scalar systems.

(3) Directly solve only the resulting implicit systems that arise due to the fast MHD waves 
that cause stiffness in the overall implicit solve; and

(4) project the preconditioned solution in characteristic variables back into the original conserved variable formulation.

A key component of this preconditioning approach is the decomposition of the PDE system into its individual characteristic components, followed by the selective solution of only those components that cause stiffness in the PDE system. In a mathematical analysis of this approach, we have shown that the accuracy is a function of both the splitting accuracy used to decompose the system, as well as the number of characteristic components chosen to precondition. The first term gives rise to an $\mathrm{O}\left(\mathrm{dt}^{2}\right)$ error term, and the latter error is dominated by the term $|\mathrm{c} \mathrm{dt} / \mathrm{dx}| /(1$ $|c \mathrm{dt} / \mathrm{dx}|)$, where $\mathrm{c}$ corresponds to the eigenvalue of the first neglected component, and $\mathrm{dt}$ and $\mathrm{dx}$ correspond to the temporal and spatial discretization parameters. Since the time step dt is selected to follow the dynamics of interest (the energetic slower wave dynamics), this estimate implies that one need only precondition those characteristics with speeds faster than the slow waves, i.e. those causing stiffness in the implicit MHD system. Experimental verification of this property is born out in Figure 1, showing that the eigenvalue distribution for the overall implicit system decreases dramatically as the number of preconditioned waves $(q)$ increases.

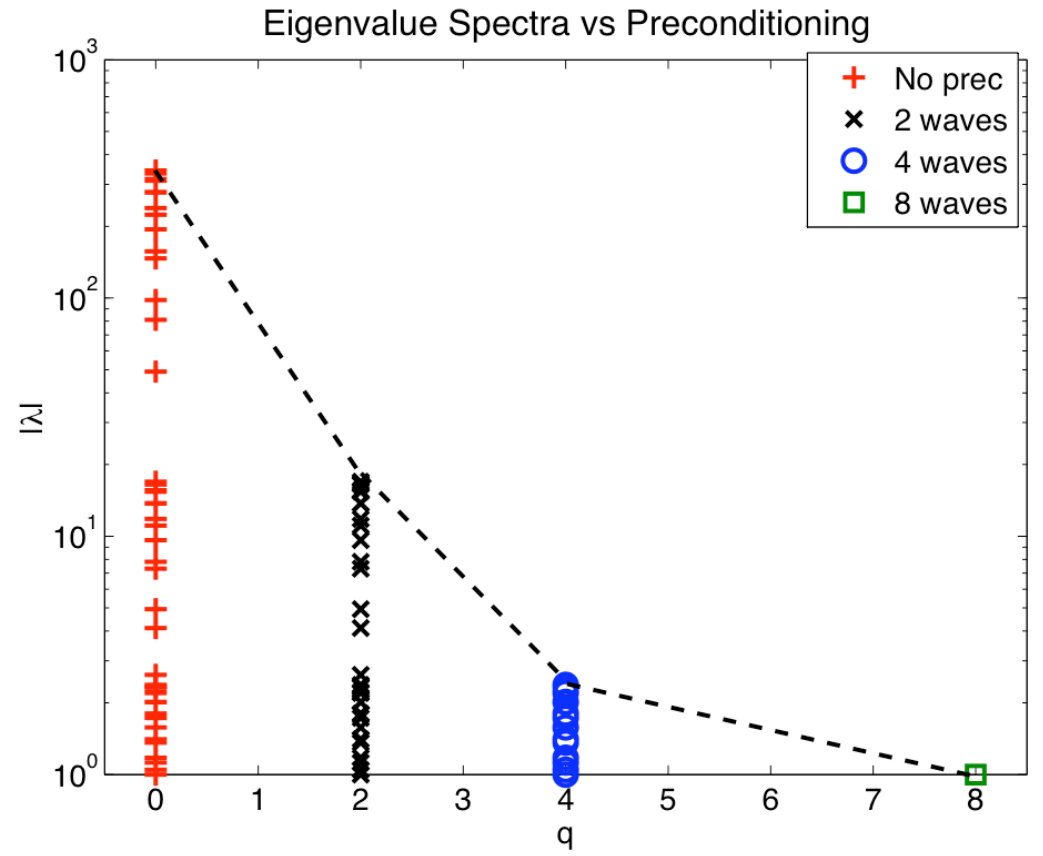

Figure 1: eigenvalue spectrum as a function of the number of waves preconditioned $(\mathrm{q})$ for an illustrative 1D MHD problem. There are 8 total waves, and as $\mathrm{q}$ increases, the preconditioned spectrum shrinks as predicted.

\section{Results:}

Initial tests on model MHD test problems (both ideal and visco-resistive) show that this approach provides both a dramatic decrease in iteration counts required for the outer iterative linear solver, along with tremendous run-time improvements over non-preconditioned implicit simulations. Specifically, testing has shown that this preconditioner performs remarkably well for problems where the underlying stiffness results from the fastest MHD waves, scales well to large problem sizes and numbers of processors (see Figure 2), and does not contribute significantly to divergence errors in the magnetic field (see Figure 3). 


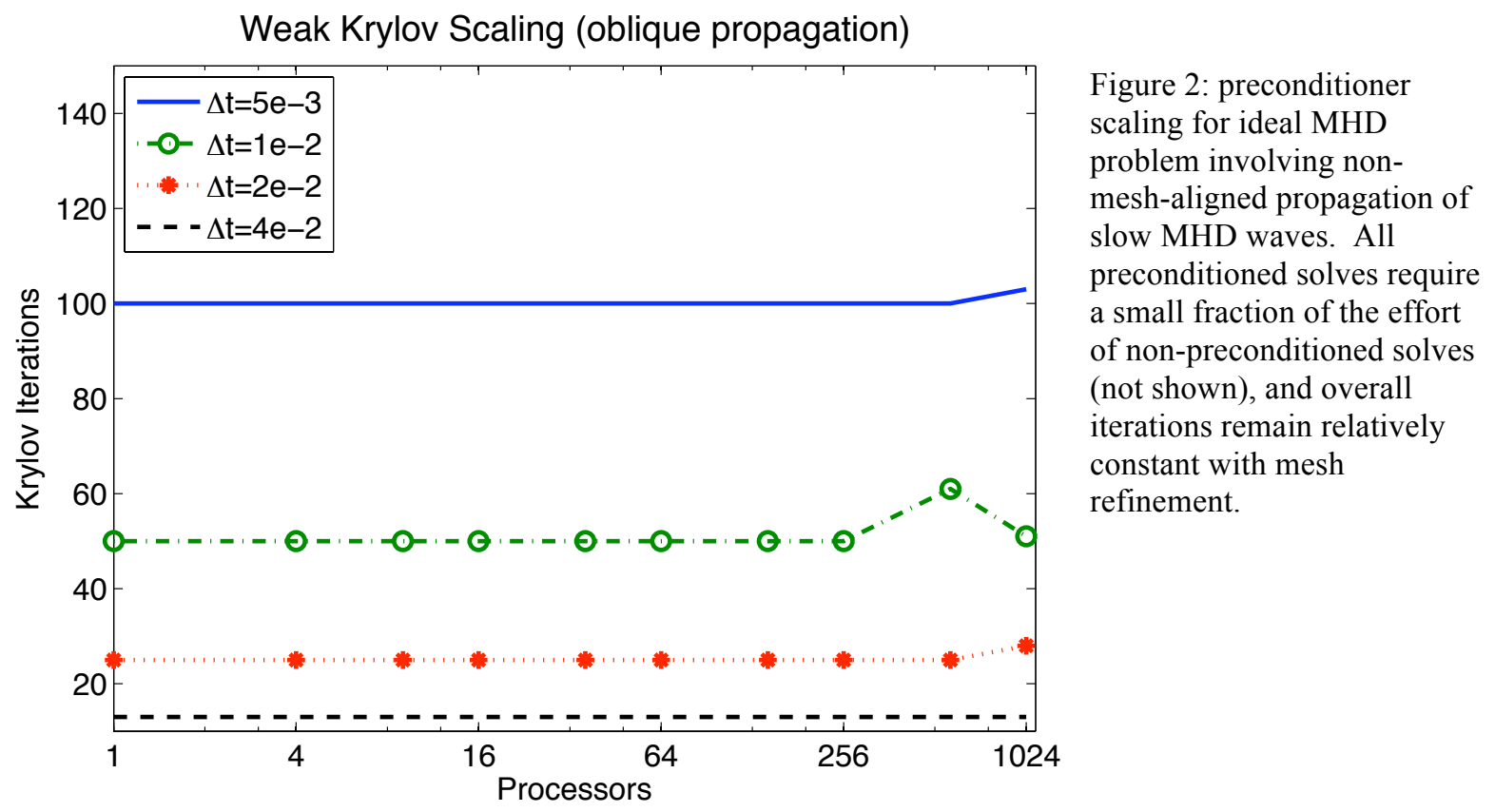

\section{Solver ramifications on solenoidal MHD constraint:}

In our studies of preconditioning approaches above, we have investigated how the choice of numerical solution methods, both at the spatial discretization level and at the nonlinear solver level, affect the underlying solenoidal constraint condition on the magnetic field. For example, it is well known that non-commutative spatial semi-discretizations can result in significant violations of this constraint, since the continuum-level identity $\operatorname{div}(\operatorname{curl}(u))=0$ inherently requires that

$$
\frac{\partial^{2}}{\partial x \partial y}=\frac{\partial^{2}}{\partial y \partial x} .
$$

Standard upwind spatial discretizations and piecewise linear finite elements violate this requirement, which gives rise to modern Constrained Transport methods and $\mathrm{C}^{1}$ finite element formulations of MHD systems. In a similar vein, we investigated whether the use of inexact Newton-Krylov methods, both with and without preconditioning, caused similar deleterious effects on the solenoidal condition.

Through numerical analysis of the inexact Newton algorithm, we found that if the underlying spatial semidiscretization is commutative, the non-preconditioned inexact Newton algorithm will exactly preserve the solenoidal constraint (to floating-point roundoff). However, when preconditioners are introduced, this theory no longer holds. However, in computational experiments of our non-preconditioned and preconditioned inexact Newton solver on standard ideal MHD test problems, we found that solenoidal violation due to preconditioning was minimal (in the $9^{\text {th }}$ digit), providing far less harm than use of non-commutative discretizations (see Figure $3)$. 


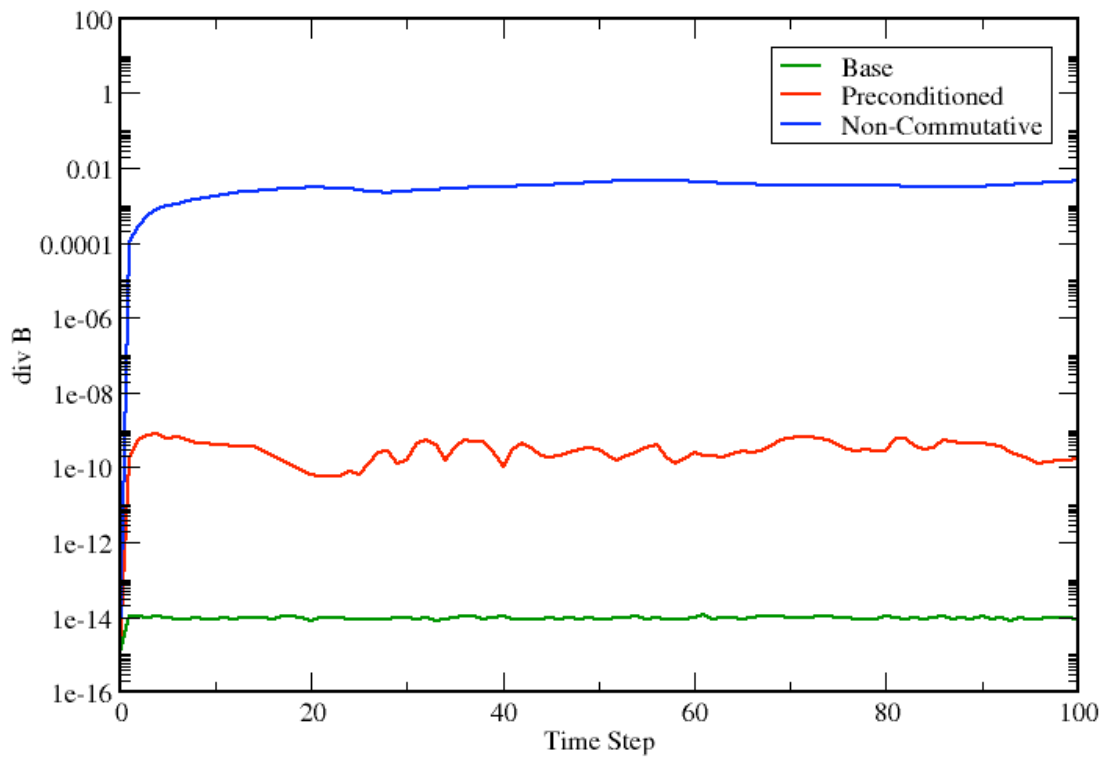

Figure 3: solenoidal constraint violations due to implicit solvers. "Base" corresponds to a nonpreconditioned Inexact NewonKrylov method. "Preconditioned" employs the fast wave preconditioner above. "NonCommutative" employs a nonpreconditioned solver, but uses an upwind spatial discretization that does not preserve commutativity in the discrete differentiation operators.

\section{Scalability improvements:}

Since completing our work on [21], we have made significant progress in improving this preconditioning approach for large-scale computation. At the heart of this preconditioner, we solve a large number of decoupled, possibly periodic, parallel tridiagonal or pentadiagonal systems. Since all other calculations in our preconditioner are spatially local, the scalability of our approach inherently relies on the scalability of the parallel periodic banded solver used at it's core; if that component solver scales well to $\mathrm{p}$ processors, the overall preconditioning approach should scale well to $\mathrm{p}^{2}$ processors for $2 \mathrm{D}$ simulations, and $\mathrm{p}^{3}$ for $3 \mathrm{D}$ simulations. In previous results [21], this inner solver was implemented using a standard Schur-complement approach, wherein each process locally solved their portion of the domain, and then a single global solve coordinated all inter-process couplings in a linear system with 2 unknowns per inter-process boundary. Due to the high degree of concurrency and significant parallel communication required for this method, tests on this component solver showed ideal parallel weak scaling only for problems using up to 16 processors in a given Cartesian direction (i.e. 256 processors in 2D, 1024 in 3D), which is clearly well below the needs of petascale computing platforms.

We therefore investigated alternate solution techniques for this inner solver for the directional preconditioner sub-systems, based on fully asynchronous execution and only requiring point-topoint communication. Tests of a new asynchronous approach, based on pipelining the large number of solves in each direction, have demonstrated near perfect weak scaling on problems using up to 1024 processors in any coordinate direction, as seen in Figure 4. As this examines only a $1 \mathrm{D}$ parallel decomposition, we estimate that this approach could result in near perfect solver scalability to up to $10^{6}$ processors for $2 \mathrm{D}$ simulations, and $10^{9}$ for $3 \mathrm{D}$. We have begun work on testing the utility of this solver on large-scale stiff ideal MHD simulations. 




Figure 4: Weak scaling of inner solvers. All processors used a base grid of $64^{3}$ cells, and we increased the number of processors in one direction. We compare three implementations: "Algorithm 1" was the method used in the publication [21], "Algorithm 2" attempted to reduce the overall number of messages, and "Algorithm 3" followed an asynchronous pipelining approach. All times are shown in seconds, and all runs were performed on the TACC Ranger system.

\section{Extensions to resistive MHD:}

In addition, we have continued development of a multigrid-based preconditioning approach for visco-resistive MHD simulations involving significant diffusive effects. While many MHD problems are dominated by stiffness due to the interaction of fast and slow hyperbolic waves, higher-order MHD models incorporate viscosity, resistivity and thermal heat conduction. Even if these diffusive terms are small (as is the case with fusion plasmas), at petascale resolutions the increased stiffness resulting from these second-order derivatives can begin to dominate the numerical convergence of an implicit solver.

This new preconditioning component employs the HYPRE linear solver library, using both their robust scalar-valued multigrid solver SMG, allowing for solutions involving anisotropic heat conduction, as well as their highly efficient PFMG solver enabling geometric multigrid solvers for systems of coupled diffusion operators. Through continued dialogue with the HYPRE solver authors, we have optimized this interface so that for large scale diffusion-dominated MHD simulations, scalability of the entire implicit MHD solver infrastructure should hinge on the ultra-scalable HYPRE linear solver components. We have begun testing of this preconditioning approach on relevant visco-resistive MHD problems.

We have also begun investigation of coupled preconditioning strategies that will employ both the fast wave stiff ideal MHD preconditioner, and the HYPRE-enabled diffusive MHD preconditioner, to allow for realistic MHD simulations in which both the stiff hyperbolic waves and the diffusion dominate various events and/or regions in the nonlinear evolution of the fusion system. These strategies rely on operator-split approaches for combining these linear preconditioning operators, in which we plan to introduce spatially- and temporally-adaptive splitting strategies to employ the appropriate preconditioning operator for the relevant physics present in the simulation.

\section{Extensions to shaped plasmas:}

In collaboration with Samtaney and Woodward, we have pursued extensions of the fully implicit, preconditioned approach to simulations of stiff, nearly ideal MHD problems posed on mapped 
spatial grids, used for representing tokamak geometries. This work is based on our previous collaborative efforts in development of fully implicit simulation technology for resistive MHD simulations using Cartesian grids [28]. Mapped spatial grids, however, allow for simulations in the native toroidal geometry of tokamak fusion devices based on logically Cartesian data structures. The use of such regular data structures then allows for highly efficient computational algorithms. Moreover, since these consist of mapped cylindrical grids, explicit-time simulations on which suffer from a well-known difficulty due to the decreasing CFL time step stability restriction due to the very small cell sizes near the core of the cylinder. Implicit methods, on the other hand, are immune to this artificial stability restriction resulting from small cells near the plasma core.

To this end, we have finished implementation of implicit simulations on mapped cylindrical grids, allowing both simulations using a fixed-step second-order time discretization, as well as a more efficient adaptive step and order time integration approach based on the CVODE solver. These extensions required bolstering of the previous interface to the SUNDIALS implicit solvers, as well as the development of robust solver algorithms that allow discontinuities arising from flux-limited spatial discretizations prevalent in high-order finite-volume algorithms. Results from these un-preconditioned implicit mapped-grid simulations of pellet injection are shown in Figure 5.

In addition, we have finished the mathematical formulation and initial development of the fastwave preconditioning approach for the mapped-grid systems. While following the same overall approach, the mapped equations induce transformations between the physical tokamak geometry and logically rectangular solver data structures, which we have incorporated into the preconditioning formulation. We are in the process of code verification for the mapped-grid, stiff hyperbolic preconditioner, and plan to begin testing of the approach on simulations of pellet injection refueling and edge-localized mode (ELM) instabilities of tokamak plasmas in the near future.


Figure 5: Implicit solver results from shaped-plasma simulation of pellet injection fueling. Left: plot of the curvilinear grid. Right: time snapshots of the density field, showing advection and ablation of the high density pellet. The implicit solver framework is inherently geometry-neutral, though the fastwave preconditioning approach relies on a logically Cartesian mesh. Here it will follow waves traveling radially (slow), toroidally (medium) and around grid-aligned flux tubes (fast), in a natural decomposition of speeds in geometric directions. 
Although implicit methods ameliorate the CFL stability restriction for such simulations, both implicit and explict methods suffer from increased spatial discretization error due to the coordinate singularity near the plasma core. We have therefore begun investigations of two additional approaches for simulations of shaped plasmas, both based on logically-Cartesian methods as in our previous work. The first is based on a level-set approach to allow for arbitrary shaped plasma geometry. The second is an embedded boundary method in which the plasma/vacuum boundary is simulated by an extremely steep gradient in density, pressure, resistivity and heat conduction in the computational domain. The first of these approaches allows a very straightforward approach for simulating the shaped plasma, but requires investigation on combining level-set and implicit-time methods. The second of these approaches allows simulations of plasma edge-core interactions including edge-localized mode (ELM) instabilities, but requires advances in scalable solver technologies for problems involving extreme anisotropies in the relevant physical parameters.

With Carol Woodward (LLNL) and her summer student, Daniel Osei-Kuffuor, we have begun studies of methods for scalable solution of highly anisotropic heat conduction processes on mapped spatial grids. Although implicit solvers for implicit heat conduction in MHD typically assume the heat conductivity to be isotropic, tokamak plasmas exhibit very high anisotropy, with the conductivity much larger along the magnetic field lines than orthogonal to them. For this work, we are investigating various multigrid methods, specifically those that have achieved high success in highly anisotropic simulations of groundwater flow. Furthermore, lessons learned from this work will directly apply to inclusion of nonlinear (and highly anisotropic) resistivity in models of fusion MHD, which is also traditionally approximated using constant resistive terms.

\section{Talks and Papers:}

Our DOE-supported work on implicit solvers and preconditioners for Cartesian MHD simulations has been published in [21-22,28]. We are currently working on a paper involving our studies of combination preconditioning combining the fast wave and diffusive solvers in [29]. We are also currently working on a paper describing our studies of implicit solvers and preconditioners for mapped-grid MHD simulations in [30].

Moreover, we have presented this work at numerous conferences, colloquia and workshops [15,8-17,19-20].

\section{Implicit Solvers for Coupled Multiphysics Simulations Involving Shocks}

\section{Problem/collaboration introduction:}

In collaboration with Doug Swesty at SUNY Stony Brook and Carol Woodward at Lawrence Livermore National Laboratory, we have continued investigations of robust and efficient approaches for fully implicit simulations of hydrodynamics and constrained hydrodynamics applications of relevance to stellar astrophysical systems. These investigations were a continuation a previous SciDAC-1 collaboration with the Terascale Supernova Initiative (TSI), 
and under SciDAC-2 were in support of a DOE INCITE project on core-collapse supernova simulations headed by Doug Swesty and Eric Myra from SUNY Stony Brook. The problem under investigation in this work is the development of fully implicit solvers for simulations of core-collapse supernovae. To properly simulate such events, models must be included for radiation transport of multiple neutrino species, hydrodynamic motion for capturing strong shocks present due to the collapsing stellar core, and chemical kinetics to model the reacting neutrino species' interactions. In such coupled simulations, the time scale for radiation transport and neutrino physics is much faster than the hydrodynamics, and traditional numerical methods handled these in a simple operator-split fashion, with the radiation transport and chemistry handled implicitly, and the hydrodynamic motion evolved explicitly. However, such splittings introduce time-discretization errors and additional stability restrictions, and the goal of our astrophysics collaborators was to instead use a fully implicit approach to allow for increased accuracy and stability.

Unlike typical physical problems tackled through implicit methods, these systems typically give rise to strong hydrodynamic shocks, incorporate non-differentiable 'switches' in their computational implementation, and involve solution components that vary by many orders of magnitude throughout a simulation, all of which result in tremendous difficulty for standard inexact Newton solver formulations. We therefore have been working toward the implicit solution of the most non-differentiable and difficult (from an implicit algorithms viewpoint) component of a fully implicit multi-physics simulation capability for such astrophysical systems, that of implicit-time shock-capturing methods.

\section{Computational approach:}

To this end, we have completed construction of a fully nonlinearly-implicit approach for hydrodynamics and gravitationally-constrained hydrodynamics evolution equations for fluid dynamics in stellar astrophysical systems. The resulting approach handles implicit formulations of both Eulerian and Lagrangian hydrodynamics, and will prove invaluable in simulations of stiffly interacting radiation-hydrodynamics, gravitational-hydrodynamics, and reactinghydrodynamics systems present in high energy and density physics applications, including problems involving strong shocks and adaptive meshes.

\section{Talks and papers:}

Details of these robustness improvements to standard methods were presented in [23], and were featured in an invited talk by Carol Woodward at the SciDAC 2008 conference. We are currently working on a more thorough article documenting and testing these approaches in detail [24]. 


\section{Implicit Solvers for Large-Scale Simulations of Cosmological Reionization}

\section{Problem/collaboration introduction:}

While not directly supported by the DOE Office of Science, we have worked to benefit leading DOE-related science applications through the use of TOPS software. The Laboratory for Computational Astrophysics at the University of California, San Diego, develops and maintains an open-source community astrophysics code (Enzo) that enables very large-scale adaptive mesh simulations of cosmological hydrodynamics, dark matter particle dynamics, and chemical ionization. This group has a long history of strong collaborations with LLNL astrophysicists and high-energy physicists with interests in large-scale simulations involving radiation and materials couplings.

\section{Numerical methods:}

In working with the LCA, we have incorporated new solvers in the Enzo code to enable fully implicit couplings between radiation hydrodynamics, chemical ionization kinetics, and gas energy feedback, with the aim of enabling petascale simulations of cosmological reionization in the early universe. This stiff implicit sub-system is solved using a customized Newton-Krylov solver infrastructure, with a Schur-complement linear solver to handle inter-physics couplings. The resulting inner Schur linear systems are then solved using a multigrid-preconditioned conjugate-gradient linear solver from the TOPS-supported HYPRE library.

In addition, this set of equations and code infrastructure serve as a testing ground for our development of methods for accurate, robust and scalable solution of coupled multi-physics systems. In order to deal with its nonlinear interaction of multi-scale processes in both space and time, we have just developed a novel approach for implicit couplings between reaction and diffusion processes. Tests of this new approach show that it provides a highly robust formulation for accurate, coupled, implicit systems of this type, even at very large time step sizes.

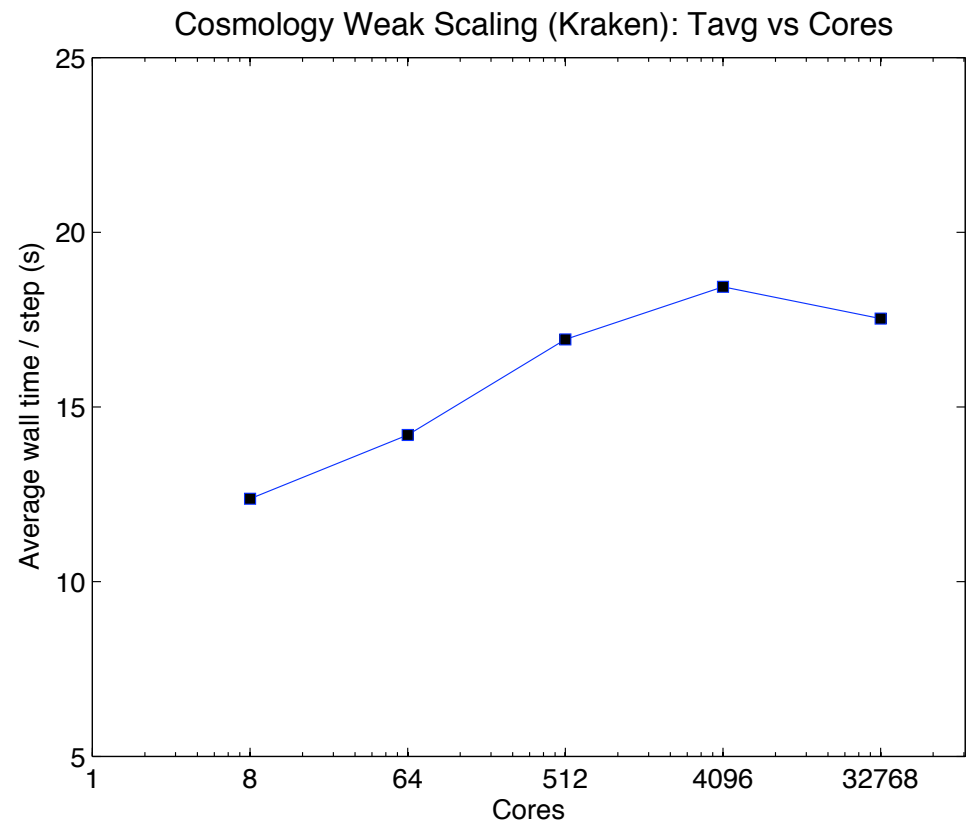

Figure 6: Weak scaling of the HYPRE-based implicit multiphysics solver for cosmological ionization. All processors used a base grid of $64^{3}$ cells, and we increased the number of processors uniformly in each direction. Note the predicted $\mathrm{O}(\log \mathrm{p})$ scaling, typical of multigrid. All runs were performed on the NICS Kraken system. The runtime decrease going to $32 \mathrm{k}$ cores resulted from dedicated "whole machine" time, in which no other uses were running jobs. 


\section{Results:}

Recent tests of this solver infrastructure have shown tremendous results on a suite of DOErelated and cosmological verification test problems, and have demonstrated near perfect weak CPU scaling to over 32000 processors on some of the largest NSF computer resources, as shown in Figure 6.

\section{Talks and Papers:}

Details of this work, including its reliance on TOPS-based software projects, are included in [2527], and have been featured in presentations [7, 18]. We are also currently working on a manuscript describing our approach for increased robustness in calculations of coupled reaction and diffusion.

In addition, much of this work has been performed in tight dialogue with LLNL radiation physicists John Hayes, Frank Graziani and Louis Howell, and may lead to strong collaborations in the near future. 


\section{Presentations, Workshops and Manuscripts Resulting From This Work}

\section{Presentations and Workshops:}

[1] Invited presentation, "Mathematical Modeling and Simulation in Fusion Energy Research", University of California at San Diego, Department of Mathematics, 2007.

[2] Invited presentation, "Implicit Integration and Constraint Preservation in Resistive MHD Fusion Modeling", University of Washington, Department of Applied Mathematics, 2007.

[3] Invited presentation, "Mathematical Modeling and Simulation in Fusion Energy Research", Duquesne University, Department of Mathematics, 2007.

[4] Invited presentation, "Implicit Integration and Constraint Preservation in Resistive MHD Fusion Modeling", Southwestern University, Department of Mathematics and Computer Science, 2007.

[5] Invited presentation, "Implicit Integration and Constraint Preservation in Resistive MHD Fusion Modeling", Tufts University, Department of Mathematics, 2007.

[6] Chair, invited plenary panel discussion, "Research Directions and Enabling Technologies for the Future of CS\&E", 2007 SIAM Conference on Computational Science and Engineering, 2007.

[7] Invited presentation, "Simulating Radiation Transport in Enzo", Center for Applied Scientific Computing, Lawrence Livermore National Lab, Livermore, CA, 2007.

[8] Invited presentation, "Modeling and HPC Challenges in MHD: Magnetically-Confined Fusion Plasmas," Young Scientists US/France bilateral workshop on Modeling and High Performance Computing," Georgetown University, Washington DC, 2007.

[9] Invited presentation, "Implicit MHD Based on SUNDIALS Software", Future Directions for M3D Workshop, Princeton Plasma Physics Laboratory, 2008.

[10] Invited presentation, "Scalable Implicit Methods for Magnetic Fusion Modeling”, Southern Methodist University, Department of Mathematics, 2008.

[11] Invited presentation, "Scalable Implicit Methods for Magnetic Fusion Modeling”, U.S. Naval Academy, Department of Mathematics, 2008.

[12] Invited presentation, "Scalable Implicit Methods for Magnetic Fusion Modeling”, Texas A\&M University, Department of Mathematics, 2008.

[13] Invited presentation, "Scalable Implicit Methods for Magnetic Fusion Modeling”, Temple University, Department of Mathematics, 2008.

[14] Invited presentation, "Applied Mathematics and Scientific Computing in Fusion Energy Modeling”, Swarthmore College Department of Mathematics, 2008.

[15] Invited presentation, "Scalable Implicit Methods for Magnetic Fusion Modeling”, Georgia Institute of Technology, Department of Computational Science and Engineering, 2008.

[16] Invited presentation, “Operator-Based Preconditioning of Stiff Waves in Implicit MHD”, 2008 SIAM Conference on Parallel Processing for Scientific Computing, 2008.

[17] Invited presentation, "Scalable Implicit Methods for Magnetic Fusion Modeling”, University of California at 
San Diego, Center for Computational Mathematics Seminar, 2008.

[18] Invited presentation, "Multi-physics/nulti-scale methods I: methods for multi-physics coupled solvers", Computational Infrastructure for Geodynamics: Workshop on Mathematical and Computational Issues in the Solid Earth Geosciences, Santa FE, NM, 2008.

[19] Invited presentation, “A preconditioned JFNK method for resistive MHD in a mapped-grid tokamak geometry", 2009 SIAM Conference on Computational Science and Engineering, Miami, FL, 2009.

[20] Invited panelist, Mathematical Formulations Panel, DOE Extreme-Scale Computing for Fusion Energy Workshop, March 18-20, 2009.

\section{Manuscripts:}

[21] D.R. Reynolds, R. Samtaney and C.S. Woodward, "Operator-based preconditioning of stiff hyperbolic systems,” SIAM Journal on Scientific Computing, 32: 150-170, 2010.

[22] D.R. Reynolds, R. Samtaney and C.S. Woodward, "Physics-based preconditioning of resistive MHD systems," (in progress).

[23] D.R. Reynolds, F.D. Swesty and C.S. Woodward, "A Newton-Krylov solver for implicit solution of hydrodynamics in core collapse supernovae," Journal of Physics: Conference Series, 125, 2008.

[24] D.R. Reynolds, C.S. Woodward and F.D. Swesty, "Efficient algorithms for implicit hydrodynamic simulation using Newton-Krylov methods," (in progress).

[25] D.R. Reynolds, J.C. Hayes, P. Paschos and M.L. Norman, "Self-consistent solution of cosmological radiationhydrodynamics and chemical ionization," Journal of Computational Physics, 228: 6833-6854, 2009.

[26] I.T. Iliev, D. Whalen, K. Ahn, S. Baek, N.Y. Gnedin, A.V. Kravtsov, G. Mellema, M. Norman, M. Raicevic, D.R. Reynolds, D. Sato, P.R. Shapiro, B. Semelin, J. Smidt, H. Susa, T. Theuns and M. Umemura, "Cosmological radiative transfer codes comparison project II: the radiation-hydrodynamic tests," Monthly Notices of the Royal Astronomical Society, 400: 1283-1316, 2009.

[27] M.L. Norman, D.R. Reynolds and G.C. So, "Cosmological radiation hydrodynamics with Enzo," in Proceedings, Recent Directions in Astrophysical Quantitative Spectroscopy and Radiation Hydrodynamics," American Institute of Physics, 2009.

[28] D.R. Reynolds, “A fully implicit numerical method for single-fluid resistive magnetohydrodynamics,” Journal of Computational Physics, 219: 144-162, 2006.

[29] D.R. Reynolds, R. Samtaney and C.S. Woodward, "Physics-based preconditioning of visco-resistive MHD systems," (in progress).

[30] D.R. Reynolds, R. Samtaney and C.S. Woodward, "Scalable implicit methods for MHD simulations in tokamak geometry," (in progress). 